\title{
Study on Driver Model Parameters Distribution for Fatigue Driving Levels Based on Quantum Genetic Algorithm
}

\author{
ShuanFeng Zhao*
}

Xi'an University of Science and Technology, School of Mechanical Engineering, 710054, Xi'an, P.R. China

\begin{abstract}
According to the assumption that fatigue study cannot reveal fatigue mechanism and nonlinear influence factors of vehicle driving closed-loop system defects, this paper proposes a driver model inversion method for studying the driver's fatigue diagnosis. Furthermore, the new method is divided into two steps: 1. By using the forecast of neural network model to build the driver-vehicle-road closed-loop model, which is adapted to the complex road conditions. Besides, and the model was used to study the changes in the closed-loop car system parameter in which the driver is in a state of fatigue. 2. By defining specific movement track through the degree of approximation of theoretical data and taking test data as the objective function, the driver parameter inverse problem was broken into multiple target optimization problems. A method of real-coded chaotic mutation of quantum genetic algorithm (GA) optimization is used to find the global optimal solution. The driving simulation test results show that under the condition of complex road conditions, the proposed algorithm in actual driving parameter inversion of the alignment is superior to the traditional genetic algorithm (GA) and the traditional quantum genetic algorithm (QGA). Finally, the relationship between pilot model parameters and fatigue factors is established.
\end{abstract}

Keywords: Fatigue driving, Quantum genetic algorithm, The driver model.

\section{INTRODUCTION}

A number of domestic and foreign scientific and technical researchers have committed to work on the techniques of driving fatigue diagnosis and diagnostic equipment, and they obtained a number of important research results. As a result, the fatigue diagnosis technology has also got rapid progress, and on this basis multiple types of fatigue testing equipment have been developed, which are applied to the engineering practice, yielding great economic and social benefits. However, the current fatigue diagnosis technology and the subsequent development of diagnostic equipment are mainly based on experience or database, such as the driving fatigue monitoring method established on the basis of the physical characteristics [1], e.g. by monitoring the facial expression feature, the pulse, heart rate changes and individual differences when the fatigue generates. In order to understand the characteristics of driving fatigue produced by the differences, if not considering the pilot of the individual, it is difficult to explain the reaction regularity of fatigue driving. However, for the whole cars' driver closed-loop system, the influence of car-driver- environment mechanism on the complex nonlinear system has not yet been fully revealed. At present, the scientific and technological workers in the domestic and foreign have carried out a lot of research by using nonlinear theory and method on the dynamic characteristics of closed-loop automobile driving system, such as the steering wheel angle signal when driving fatigue appears in chaos [2],

*Address correspondence to this author at the Xi'an university of Science and Technology, School of Mechanical Engineering, 710054, Xi'an, RR china; Tel: +86-029-85583055; E-mail: zsf@xust.edu.cn the closed-loop automobile driving system in the road environment, graded and mutation research [3] and so on.

All these have made a series of important research results based on automobile driving and driving fatigue diagnosis and prediction. Also, it provides certain theoretical basis. But there are still many important questions remaining to be solved, of which the two most important are: 1 . The build of applicable multi-factor coupling driver model; 2 the identification of pilot model parameters, that is to say: whether the parameter of automobile driver's fatigue can be calculated through analyzing the operation of the driver action and movement parameter.

In the process of solving the two key questions, the results of the pilot model conclude the following aspects: In 2002, Dario et al used the theory of ACT - R on the driver's behavior to forecast modeling [4]. Academician of the Chinese scholars, Guo Konghui made the curve models namely, the optimal control model and the optimal spline [5]. In the aspects of parameter identification on closed-loop driving system, literature [5] assumes that the pilot model uses a driving simulator under the condition of the simple path, and uses the global evolution in the local optimization identification algorithm to identify the driver model parameters.

This paper will analyze the actual characteristics of the closed-loop system of the car-driver-environment. Besides, a model has been designed which could reflect the path in the process of driving, external traffic conditions (environment), vehicle performance as well as the driver's role is considered as the core to establish this people-centered closed-loop drive system model under complex road conditions. In the process an algorithm has also been proposed for the driving 


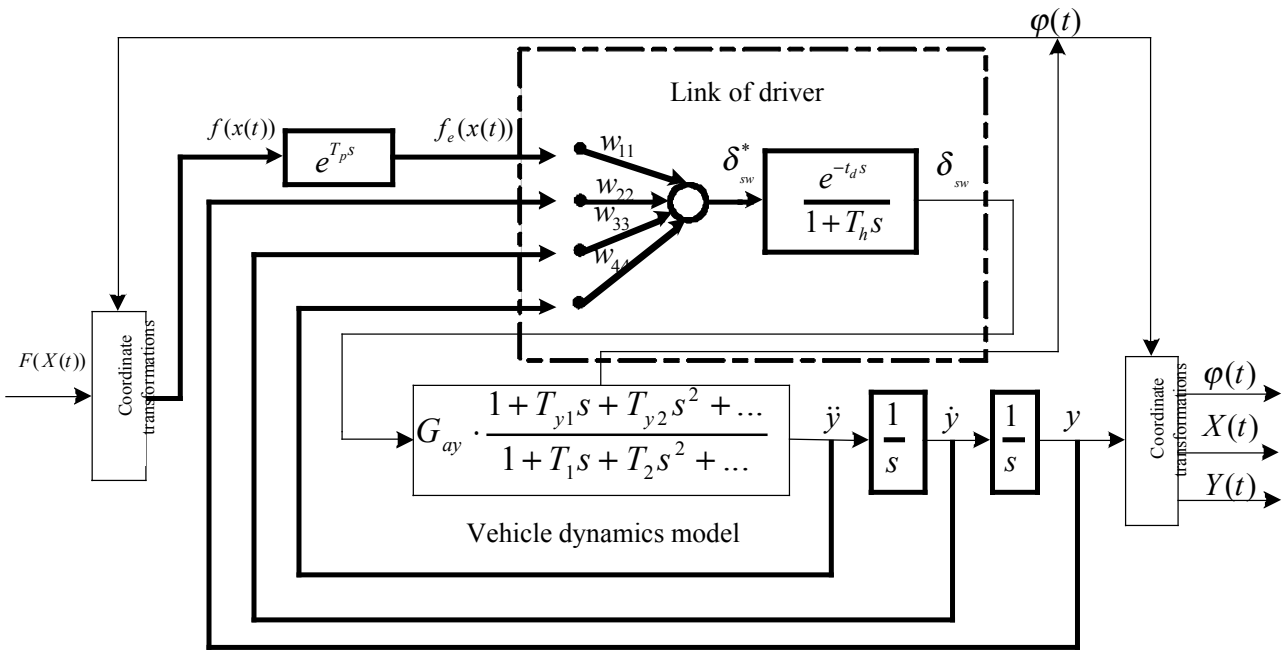

Fig. (1). The basic network aiming to optimize the basic diagram of artificial neural network.

parameters identification based on real-coded quantum genetic algorithm, so that the driver parameters can be recognized.

\section{THE CLOSED-LOOP DRIVING MODEL UNDER THE COMPLEX ROAD CONDITIONS}

Fig. (1) shows the basic network aiming to optimize the basic diagram of artificial neural network, and its physical meaning is: $F(X(t))$ is the actual path in the current time of the absolute coordinates as well as the way of absolute coordinates information through coordinate transformation, and thus becoming a track vehicle in the way of relative coordinates. Through an aiming device (transfer function $e^{T_{p} s}$ ) and according to the orbit $f(x(t))$, the instantaneous state of the vehicle $y(t)$ and the road ahead $\dot{y}(t)$, there will be an optimal steering wheel angle. By using the pilot model with the various input variables through the artificial neural network between neurons and superposition of the comprehensive transfer function, the pilot will be simulating to determine the optimal process of the steering wheel angle. Considering the fact that the actual car driver is an ideal system, which is inevitable to avoid hysteresis characteristics. Thus there is lagging link $e^{-t_{d} s} /\left(1+T_{h} s\right)$, of which $T_{h}$ is the lag time of driver operating actions, $e^{-t_{d} s}$ is the nervous system reaction lag of the driver, $t_{d}$ is lag time of neural response, $\delta_{s w}$ is the actual steering angle of the driver.

$V(s)=G_{a y} \frac{1+T_{y 1} s+T_{y 2} s^{2}}{1+T_{1} s+T_{2} s^{2}}$

$T_{c}=t_{d}+T_{h}+T_{1}-T_{y 1}-a T_{p} / 3$

$V(s)$ represents the vehicle model as well as it is the second order transfer function of the vehicle lateral acceleration $\ddot{y}$ and the steering wheel angle $\delta_{s w}, y$ is lateral dis- placement response. $G_{a y}$ is the lateral acceleration of the steady state gain; $T_{y 1}, T_{y 2}, T_{1}, T_{2}$ are some constants; $a$ is the followed-order number.

From Fig. (1), the model of pilot to control the direction of the system (empty boxes) is a multi-input single output system. The input are instant state $y(t), \dot{y}(t), \ddot{y}(t)$ of the vehicles following the expected trajectory and the output is the vehicle's steering angle $\delta_{s w}$. In the process of actual driving, due to the fact that the nature of people is influenced by physiological and environmental factors such as psychological change over time, the measured data includes the change of steering wheel angle, namely driving disturbance. Generally speaking, the randomness of driving perturbation can be thought of as white noise process. But different drivers driving disturbance in the driving process is different, and the disturbance amplitude is small, which can be noticed in the parameter identification during the process of measurement. Due to the fact that the advanced test and processing technology are used to ensure the measurement of the noise signal which is small, and it is due to white noise process, so that the random disturbance mainly displays the driver behavioral characteristics of uncertainty in the process of driving changes. The input and output data presented in Fig. (1), such as vehicle's driving time $t$, expected trajectory $f(t)$, the actual steering angle $\delta_{s w}$, lateral acceleration $\ddot{y}$, lateral velocity $\dot{y}$, lateral displacement $\dot{y}$, can be measured by passing the driving simulator test. Besides, its measurement precision is higher.

\section{THE IDENTIFICATION OF DRIVER MODEL PA- RAMETERS}

The driver is the crucial link in the closed-loop system of people-car-road. Thus, an advance optimization of artificial neural network was carried out based on identification of the key parameters of driver model, which actually belong to the problem of closed-loop system parameter identification. Ac- 


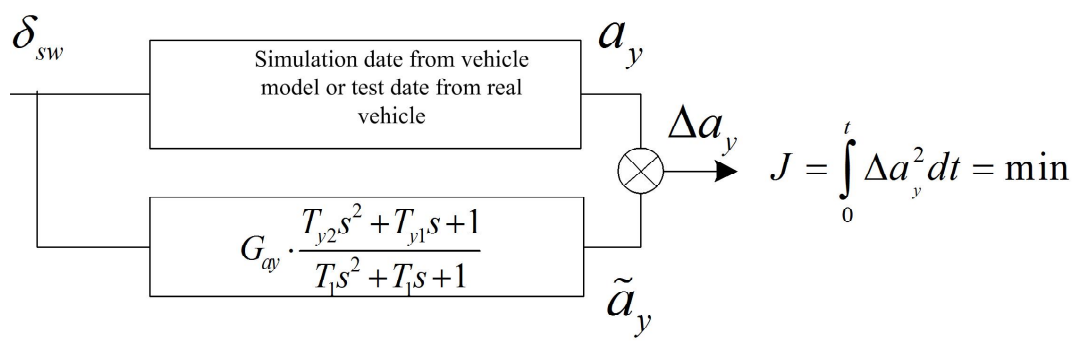

(a) The block diagram of vehicle model parameter identification

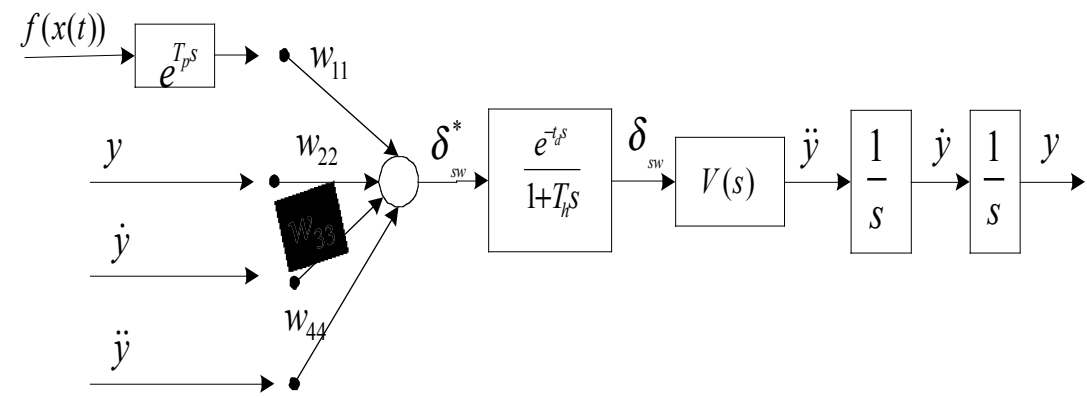

(b) The block diagram of driver model parameters identification

Fig. (2). The diagram of cars' closed-loop system parameter identification.

cording to the closed-loop system identification theory, if the input/output subsystems of the closed-loop system are measurable, the suitable method of open-loop identification can be adopted. The measurement of the input and output of the subsystem model is set up directly (direct identification method). At present, the model parameter identification method is usually divided into frequency domain and time domain method: the frequency domain method is mainly through Fourier to transform the original data. And input and output transform exist in frequency domain, and then the frequency response of the system is to simulate nonlinear parameters and the characteristics. Its precision mainly depends on the estimation and precision of the frequency characteristics. The basic idea of the time domain identification implies using the finite difference model ARMA (autoregressive moving average) equation for least squares identification of input and output data, and then do $Z$ transform to define transfer function for a discrete system. In this paper, in the time domain, it is by using quantum genetic algorithm that the global evolutionary optimization method and identification of parameters for model of direct control over the pilot are realized. There is neither a need for changes in frequency domain, nor deviation from transformation to improve the precision of parameter identification accuracy. More importantly, parameters for the natural driver are identified to involve in the operation of the object driver vehicle model.

Because the actual vehicle model (or driving simulation) tends to be very complicated due to the unavailability of the the software for the mathematical description model of vehicle. As a result, it will not be able to directly calculate the pilot model parameters, which are needed for the vehicle model. So before applying the pilot model, it is necessary to simplify the complex vehicle model. Also, since the complex vehicle model is equivalent to two degrees of freedom linear vehicle model, according to the theory of system parameter identification method, the pilot model which is needed for vehicle kinetic parameters identification of the model can be calculated.

Fig. (2) illustrates the vehicle model parameter identification block diagram and its specific identification process is as follows: select a typical vehicle steering wheel angle signal $\delta_{s w}$ (or a set of test data of the vehicle) then input it to the vehicle model of complex system, with lateral acceleration $a_{y}$ of the vehicle as the output of the system. And tie the same steering wheel angle, then input to the equivalent of two-degrees-of-freedom vehicle model, the output is the lateral acceleration $a_{y}$, and the difference between $a_{y}$ and $a_{y}$ is taken as objective function, so as to optimize the parameters of vehicle model.

According to the static conditions based on literature [6], the equation can be shown in Fig. (1) in which driver closedloop optimization model of artificial neural network is converted into open-loop form, as shown in Fig. (2b).

The relationship between advanced pilot model and the weights of optimized neural network is not isolated. On the contrary, there is a certain link between time lag link with the driver, car and structural parameters. By combining the neural network model under the static condition with [3], the relationship between the driver motor closed-loop system and the parameters can be obtained.

$\left\{\begin{array}{l}w_{11}=-w_{22}=1 \\ K_{0}=T_{p}^{3} /\left(6 \cdot T_{q 1}\right)\end{array} \quad w_{33}=-T_{p} \quad w_{44}=K_{0}-T_{p}^{2} / 2\right.$

From equation 3, it is shown that there is close relationship between pilot model with the values, parameters $K_{0}$ 
and the driver's time lag link $T_{q 1}$ (including the driver model and the structural parameters of the vehicle information). Among them, the relationship between driver model parameters $T_{q 1}$ and $T_{q 2}$ is as follows:

$$
\left\{\begin{array}{l}
T_{q 1}=T_{h}+t_{d}+T_{1}-T_{y 1} \\
T_{q 2}=T_{h} \cdot T_{1}+\frac{t_{d}^{2}}{2}+\left(t_{d}-T_{y 1}\right) \cdot\left(T_{h}+T_{1}-T_{y 1}\right)+T_{2}-T_{y 2}
\end{array}\right.
$$

Of the equation $4, T_{h}, t_{d}$ are the time constants of the pilot model lag; $T_{1}, T_{2}, T_{y 1}, T_{y 2}$ are the parameters of the two degrees of freedom vehicle model.

When the driver model parameters are identified, the open-loop driver model and vehicle model are close to theoretical data, and the corresponding test data shows the optimization of objective function. Therefore, setting the optimization goal is essential for avoiding the steering wheel angle error, for the sum of squares of lateral acceleration error, for the error sum of squares, for the error sum of squares of lateral displacement and the weighted values of the three $J_{E}$.

$J_{E}=w_{1} \cdot J_{e 1}+w_{2} \cdot J_{e 2}+w_{3} \cdot J_{e 3}$

Of the equation: $J_{e 1}=\int_{0}^{t}\left(\delta_{s w}-\hat{\delta}_{s w}\right) d t$ is the steering wheel Angle error indicator; $J_{e 2}=\int_{0}^{t}(\ddot{y}-\hat{y}) d t$ is the lateral acceleration error indicator; $J_{e 3}=\int_{0}^{t}(y-\hat{y}) d t$ is the lateral displacement error indicator; $\delta_{s w}, \ddot{y}, y$ are the experimental data of a driving simulation experiments. $\hat{\delta}_{s w}, \hat{\ddot{y}}, \hat{y}$ are collected for open-loop driver model and vehicle model to calculate the theoretical data. Therefore, $w_{1}, w_{2}, w_{3}$ are the weighted values, respectively, $0.8,0.1,0.1$.

\section{QUANTUM-INSPIRED GENETIC ALGORITHM}

\subsection{Chromosome Encoding and Decoding}

Encoding and decoding are the key factors in using genetic algorithm to solve the problem. It is also realized that the pilot model parameters identification is the key to optimize of the objective function. It is necessary to establish all points in the problem space of chromosome (candidate); Chromosomes in the GA space correspond to all the problems of candidate solution space: i.e. chromosomes and their candidate solution. Because the optimization problem is involved in the solution space dimension, the adopting qubit chromosome will take place of traditional chromosome. In adopting the Qubit coding method, a qubit chromosome can represent superposition state. Compared with the traditional genetic algorithm, the new alogrithm has better diversity of population [7].

The quantum chromosome can be defined as follows: $\left(\begin{array}{cccc}\alpha_{1} & \alpha_{2} & \ldots & \alpha_{m} \\ \beta_{1} & \beta_{2} & \ldots & \beta_{m}\end{array}\right)$

Among them, $\left|\alpha_{i}\right|^{2}+\left|\beta_{i}\right|^{2}=1, i=1,2, \ldots m$ and the corresponding phase can be expressed as $\phi_{i}=\arctan \left(\beta_{i} / \alpha_{i}\right)$.

Considering the problem of main analytical function of numerical optimization, real number chromosome coding is used here. Different from the traditional way of QGA coding, real quantum coding adopts one qubit rather than multiple qubits to express a chromosome information. Also, by using the method of chaos variable and the qubits in evolution of crossing probability, the coherence of quantum bits and chaos characteristics is fully used. The real quantum coding can be expressed as follows:

$P(t)=\left(\begin{array}{cccc}x_{1}^{t} & x_{2}^{t} & \cdots & x_{N}^{t} \\ \theta_{1}^{t} & \theta_{2}^{t} & \cdots & \theta_{N}^{t}\end{array}\right)$

Among them, $x_{i}^{t}, i=1,2, \ldots N$ is subject to the uniform probability $x_{i}^{t} \in\left(a_{i}, b_{i}\right)$ distribution. $\theta_{i}^{t}$ is the first variable phase angle of the chromosome, whose formula (7) can be expressed as follows:

$\theta_{i}^{t}=\arcsin \left(\frac{x_{i}^{t}-a_{i}}{b_{i}-a_{i}}\right)$

\subsection{The Fitness Function and Genetic Operation}

(1) Fitness function. When apply the evolutionary algorithm for the identification of driver's parameters, a pair of driver parameters is built to evaluate the judging function, because the optimization goal is to find parameters to minimize the total error indicators $J_{E}$. However, the quantum evolutionary operation is based on maximum adaptation. The fitness function value chosen here is: $f(x)=1 / J_{E}$.

(2) The selection strategy. In this paper, the niche coevolution strategy based on probability partition and the optimal method to choose the individual, have been adopted. The heart of the synergy evolution strategy is the qubits probability space, which is divided into the formation of population $N_{\text {iche }}$ and initialization $N_{\text {iche }}$. The probability space test can be carried out in accordance with the equation (9), while quantum gate updates the algorithm within each child population evolution. When it is in global migration conditions, keep the best individual to the global optimum; When it does not meet the global migration conditions, use the global optimum guidance for groups and individuals to update quantum gate.

$$
\left(\begin{array}{c}
\alpha_{k} \\
\beta_{k}
\end{array}\right)=\left(\frac{\sqrt{k / N_{i c h e}+\delta}}{\sqrt{1-\left(k / N_{\text {iche }}+\delta\right)}}\right)
$$


Equation $N_{\text {iche }}$ is the number of niche subgroup, $\delta$ is the limit probability of chromosome numbered 0 or $1 . \mathrm{k}$ is for the serial number of niche.

(3) Crossover and mutation. If we suppose that the generation of the best individual and its phase angle is $B(t)$ and $\theta(t)$ respectively, the corresponding population are $\left\{P_{1}(t), P_{2}(t), \cdots, P_{n}(t)\right\}$, and its phase angle is $\left\{\theta_{1}(t), \theta_{2}(t), \cdots, \theta_{n}(t)\right\}$. We get the next generation by adopting the method of cross probability.

$$
\begin{aligned}
& P_{i}(t+1)=B(t) \cdot \cos ^{2}\left(\Delta \theta_{i}\right)+P_{i}(t) \cdot \sin ^{2}\left(\Delta \theta_{i}\right) \\
& \Delta \theta_{i}(t)=\theta_{i}(t)-\theta(t), \quad 1 \leq i \leq n
\end{aligned}
$$

In this paper, chaotic sequence disturbance is used for the current generation of all real numbers in the chromosomes of the angle of phase-control clipping. The generation of chaotic sequence logistic mapping is used to get the chaotic sequence $\mathrm{C}$, and the amplitude adjusts itself in accordance with the adaptive value. Then, the corresponding number $\Delta \theta_{i}$ can be obtained:

$$
\Delta \theta_{i}=e^{\frac{b_{i}-f\left(x_{i}\right)}{b_{i}}} \cdot C(i)
$$

By using (12 equation) the revolving door of quantum genetic algorithm, the variation of population individuals can be realized.

\subsection{Algorithm Steps}

The concrete implementation steps are as follows:

Step 1: Initialization: Determine the population size $n$, population $N_{\text {iche }}$, maximum algebra $G n_{\max }$, limit probability $\delta$ and convergence judgment rule. In each child's solution space, by the use of uniform distribution random function, we get a real chromosome $P(t)$ and the initial population. And by using the formula (7), we can get the phase angle of each chromosome $\theta$.

Step 2: With the evaluation function, the evaluation of of all population individual fitness can be obtained. Furthermore, the best individual migration can be saved within each species.

Step 3: Reserve the best individual and its corresponding phase angle $\theta$, then judge whether it can meet the termination conditions, or it is in line with the global optimal conditions for retention of the global optimum. Otherwise, we take the step (4).

Step 4: For child populations, we applied formulas (10) and (11), to improve the population crossed to produce a new generation of population $P(t+1)$. For the corresponding individual with weaker fitness, we used formula (12).

Step 5: Mutation of the evolution algebra 1, while algorithm goes to step (2). This process does not stop until the algorithm meets the requirements or execution of the precision genetic.

\section{TEST ANALYSIS}

This research makes full use of the car driving simulator to carry out the people-car-road and environmental closedloop system experiment. Driving route takes the complex road environment, including a smooth road and a large curvature curve. Three different skilled fatigue driving workers take part in the test and the speed limit is $v=0 \sim 40 \mathrm{~m} / \mathrm{s}$. By adopting the cognizance of driver's mental fatigue method, we use signals and the method of literature [8] for objective evaluation. Thus, fatigue state is divided into the non- fatigue state, slight fatigue state, and moderate fatigue state. In the experiments, we use models by altering six parameters which influence the stability of vehicle operation (center of mass position, wheels cornering stiffness, steering system stiffness, coefficient of rear axle tilt, steering transmission ratio and the rotational inertia of the vehicle around the vertical axis). Then, we got 14 different deformation car record data, including road input $f(x(t))$, the steering wheel angle $\delta_{s w}$, lateral acceleration $\ddot{y}$, lateral velocity $\dot{y}$, lateral displacement $y$, the forward speed $v$, etc. It is worth noted that the changes in the velocity of the car will affect automobile dynamic characteristics. And since the general case study of a coupled system is complex, it is expected that in the project complex system can be decoupled into several small and independent subsystems. In fact, within the pre at time (less than 2 seconds), the vehicle's actual longitudinal velocity changed a little. However, in this short period of time, its influence can be ignored. According to the vehicle's dynamics model, the $G_{a y}$ in equation (1) can be represented as follows:

$G_{a y}=\frac{u_{a}^{2}}{l \cdot\left(1+K \cdot u_{a}^{2}\right)}$

From the above equation, it can be seen that there is a function between the vehicle longitudinal velocity, lateral acceleration of the steady state $G_{a y}$ and the steering wheel angle. Furthermore, the greater the vertical speed is, the greater the steady state lateral acceleration that is used to measure the steering wheel angle. As a result, a module has been described in this article, which is necessary to output the longitudinal velocity rate feedback to the pilot model. Thus, the dynamic characteristics of size may vary with the dynamic change of longitudinal velocity so that they cannot only be more accurate in complex situations but also can identify the pilot model parameters.

In the driver parameter identification module and the optimization module of the quantum genetic algorithm (RCQGA), the real-coded chaotic mutation and the initialization parameter respectively, are set to: $n=6, N_{\text {iche }}=15, G e n_{\max }=500, \delta=0.017$. We also take the result of driving simulator record data for the driver module parameter identification and the optimization method. The basic quantum genetic algorithm (QGA) and genetic algorithm (GA) were analyzed respectively. The results show that by using real-coded chaotic mutation of quantum genetic 


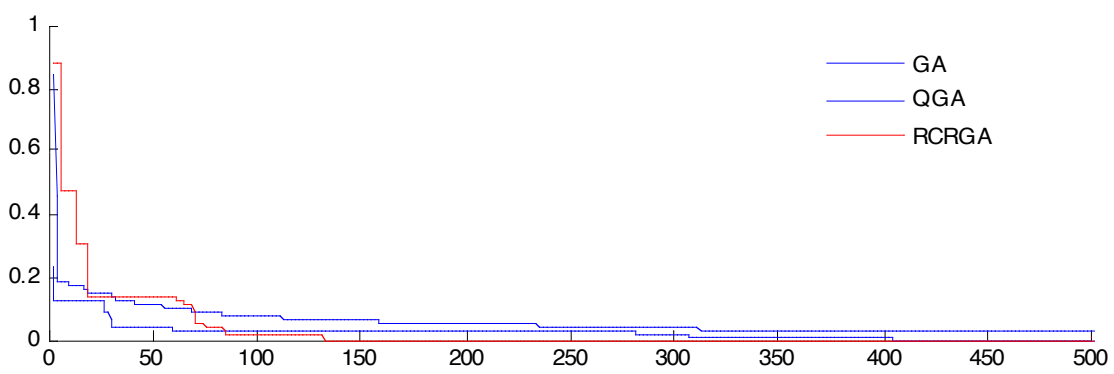

Fig. (3). The relationship between the evolution algebra and index.

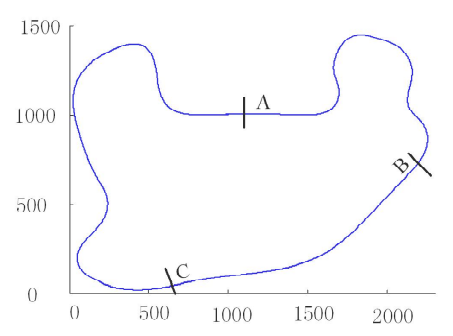

( a ) virtual road test

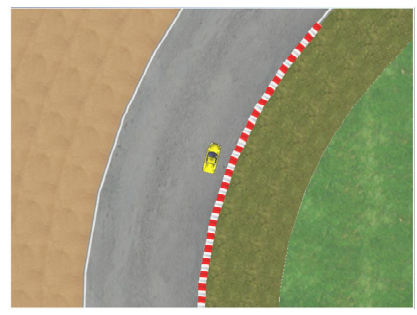

(c) Overlooking form B point

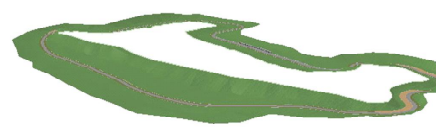

( b ) Road path overview

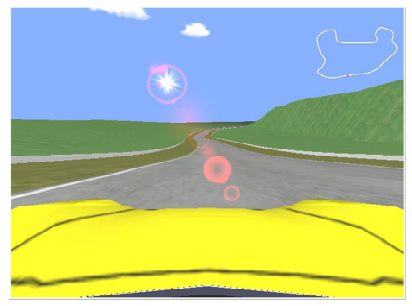

(d) Driver's seat camera at $\mathrm{C}$ point

Fig. (4). The driving simulation test paths.

algorithm, we can identify the fact that the parameters and actual driving simulation matches are the highest, so is the orbit identification model with high accuracy.

Fig. (3) illustrates a different optimization algorithm of iterative algebra and the relationship between index error. We can find that RCQGA optimized iterative is the least and convergence property is best. Fig. (4) shows the driving simulation test paths.

Fig. (5). shows the contrasting curve between car driving route in closed-loop driving experiments and parameter identification of the theory. The points on the graph line represent road center line, while the solid lines represent the car's actual movement track line. By using the theoretical identification of parameters and theoretical calculation of the trajectory path of experimental road, and by using complex stretching of road grade into small and big curvature sections, we simulated the actual driving in the process of the different influence of a road camber on drivers' mind. The zoom graph shows in detail that by using the theory of RCQGA, the parameters identification help to calculate trajectory and the movement track of car that almost overlap. All these illustrate the correctness of the driver parameter identification method.

Fig. (6) shows a parameters model in which different levels of drivers fatigue both in the graded road section (Fig. 6a) and large curvature road section (Fig. 6b) are given. And the distribution is $T_{p}, T_{h}, T_{d}$. Based on driver fatigue degree, 14 different kinds of driving models under complex conditions are given (as shown in Fig. 4). The experiment is repeated for 10 times, and the driver's driving model parameters are identified through collected data. The identification results are described in the form of three-dimensional space distribution. And the results will be used to analyze various parameters of the object driver and the standard deviation of the average statistics. The first analysis is carried out under normal driving conditions and based on the influence of the driver's parameters. From the contrast shown in Figs. (5a, b), it can be seen that in the case of normal driving, big road curvature has some influence on the driver's nervous system. Besides, the response time $t_{d}$ is greater than the reaction time under the condition of graded road, suggesting that when the driver is in an unfamiliar road environment, it requires some thinking time. Besides, under the familiar road environment, driver almost subconsciously speed up in the case of large curvature road, so that drive time $T_{p}$ will be smaller than unfamiliar road environment design time. The conclusion conforms to the driver's actual situation. So, from another aspect, it illustrates the correctness of the driver parameter identification method. 


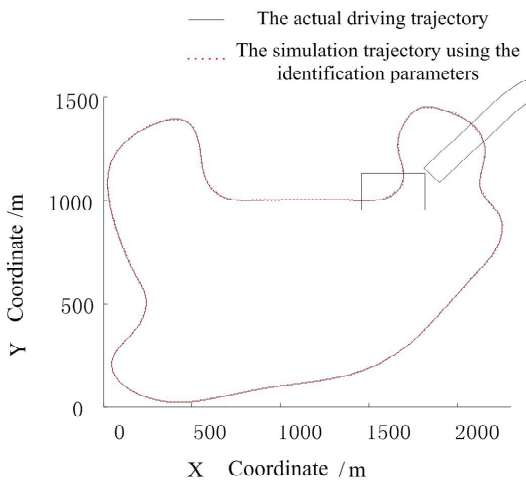

(a ) driving trajectory

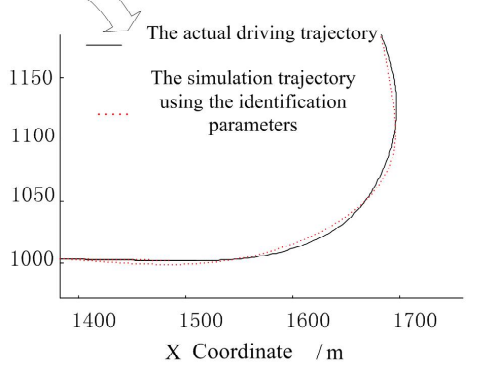

(b ) Zoom

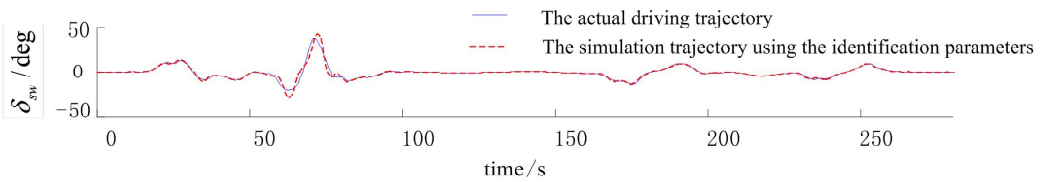

(c ) The steering wheel angle The actual driving trajectory

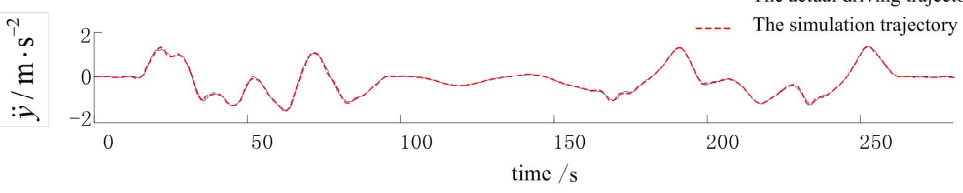

(d) The lateral acceleration

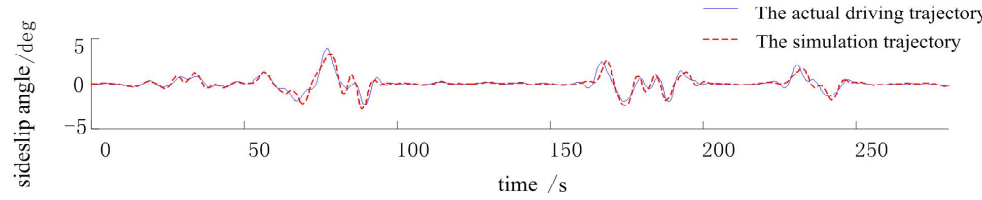

(e ) The vehicle sideslip angle

Fig. (5). The contrast between normally driving theoretical data and actual data.
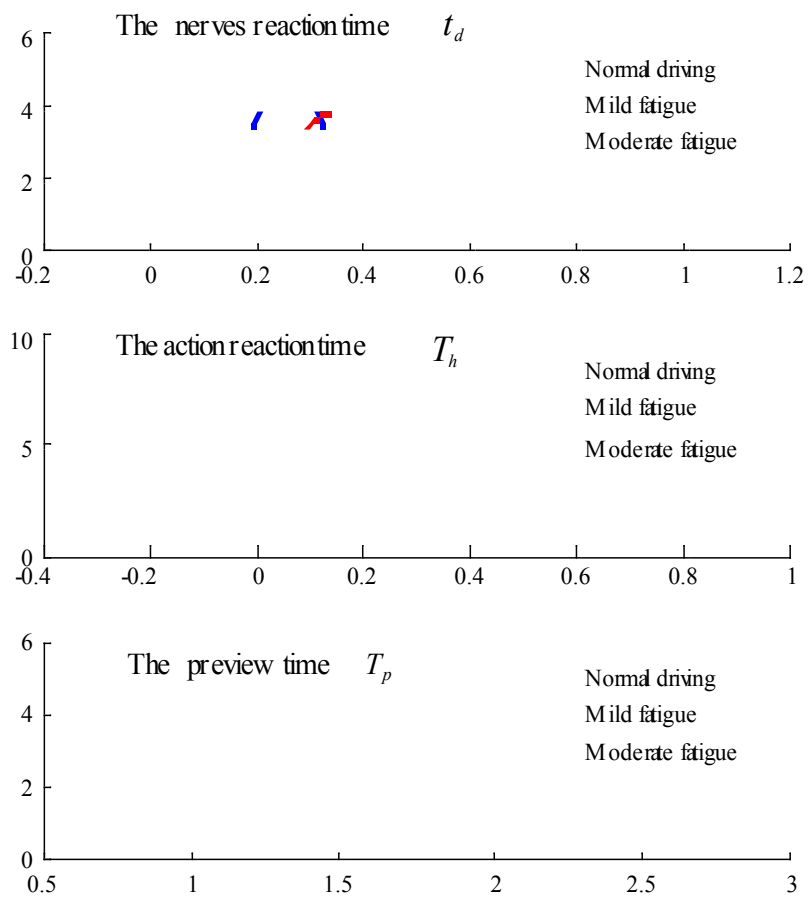

(a) Graded road segments

Fig. (6). The driver model parameters in different distributions and different fatigue driving levels. 
Through comparative analysis of different fatigue states as well as based on various driver parameters under different curvatures, we found that a driver's driving under the normal state on graded road's segments is relatively concentrated in the graphical distribution compared to the skilled driver's performance. The change in the parameters is more stable and the standard deviation is small. Besides, the evaluation function of the population variance is small when the driver is in fatigue state. And with each parameter, the range is larger, the standard deviation is larger, so is the target population variance. It indicates that the driver, who is in the large curvature road environment, has a more concentrated spirit to a certain extent. Although drivers can resist fatigue, there are certain differences in the length of the reaction time. It also suggests that in the condition of fatigue driving, a high graded curvature environment is more prone to accidents.

\section{CONCLUSION}

In this paper, we used an optimized driver model of artificial neural network and the model of closed loop vehicle system under the complex road conditions. And then according to the static condition [6], the driver closed-loop model, which is conducive to the pilot, is converted into open-loop form for parameter identification. On the driver parameters identification, this paper puts forward an algorithm using real-coded chaotic mutation of quantum genetic algorithm (RCQGA) for driver parameter identification in time domain. It must be pointed out that this method does not need to be transformed or improved for judging the precision of parameter identification. Compared with the traditional QGA algorithm and GA algorithm, RCQGA coding algorithm is convenient, time-saving, and with a fast convergence time, have the advantages of global optimization ability. As a result, it is suitable for the solution of the problem. The identification results show that the theoretical value and experimental value are consistent. According to the driver model parameters, it can better express the driver's driving behavior in a short period of time (the pilot fatigue status has not changed). The identification results show that under the condition of graded curvature and normal driver, each parameter changes smoothly. Besides, range of each parameter of the driver fatigue state is larger. On the large curvature roads and under the condition of fatigue, the driver who is under various fatigue parameters range, did not differ significantly. However, it is worth noted that the length of the reaction time and its parameters have a certain difference. On one hand, it suggests that under the condition of fatigue driving, high graded curvature environment is more prone to accidents, which is identical with our daily life experience. On the other hand, it illustrates the correctness of the method proposed in this paper. Besides, the method can not only provide a basis for further study of vehicle road closed-loop system, but also a new theoretical support for the research based on driving behavior and the state of the vehicle driving fatigue.

\section{CONFLICT OF INTEREST}

The author confirms that this article content has no conflict of interest.

\section{ACKNOWLEDGEMENTS}

This work is supported by National Natural Science Foundation of China (Project 51305339) and Natural Science Basic Research Plan in Shaanxi province of China (Program No 2013JM7022).

\section{REFERENCES}

[1] S.K.L. Lal, A. Craig, P. Boord, L. Kirkup, and H. Nguyen, "Development of an Algorithm for An EEG-based Driver Fatigue Countermeasure," Journal of Safety Research, vil. 34, pp. 321-328, 2003.

[2] Y. Takei, and Y. Furukawa, "Estimate of Driver's Fatigue through Steering Motion," In: Proc. of IEEE International Conference on Systems, Man and Cybernetics, vol. 1pp. 1765-1770, 2005.

[3] L. Fletcher, L. Petersson, and A. Zelinsky, "Driver Assistance Systems Based on Vision in and out of Vehicles," In: Proc. of the IEEE Intelligent Vehicles Symposium, pp. 322-327, 2003.

[4] D.D. Salvucci, E.R. Boer, and A. Liu, "Toward an integrated model of driver behavior in a cognitive architecture," Transportation Research Record, 2001.

[5] J. Cao, H. Lu, K. Guo, and J. Zhang, "A driver modeling based on the preview-follower theory and the jerky dynamics," Mathematical Problems in Engineering, Article ID 952106, 2013.

[6] D. Haitao, K. Guo, W. Fang, J. Cao, and T. Yang, "An Analytical Driver Model for arbitrary Path Following at Varying Vehicle Speed," IJVAS, 2004.

[7] S. F. Zhao, G. Xu, T. Tao, and L. Lin, "Real-coded Chaotic Quantum-inspired Genetic Algorithm for Training of Fuzzy Neural Networks," Computers \& Mathematics with Applications, vol. 57, pp 2009-2015, 2009.

[8] C. Zhang, C. Zheng, X. Pei, and Q. Yi, "Power Spectrum Analysis on the Multiparameter Electroencephalogram Features of Physiological Mental Fatigue," Journal of Biomedical Engineering, vol 26, pp. 162-168, 2009. 